\title{
Estabilidad y funcionalidad de sellantes dentales en altas temperaturas ambientales
}

\author{
ANASTASIA MISHINA, ROSA MAYELÍN GUERRA, IVETTE DURÁN RAMOS, JUAN D. SABATIER \\ Aseguramiento de la Calidad, Centro de Biomateriales, Universidad de la Habana, Cuba
}

\begin{abstract}
Resumen
Los sellantes dentales son materiales que se emplean en estomatología para sellar las fosas y fisuras oclusales de los dientes y evitar la adherencia del estreptococus mutans agente que produce la caries dental. Estos materiales se suministran en dos partes que al mezclarse producen el endurecimiento de la resina. Sin embargo, estos productos presentan dificultades para su comercialización en los países tropicales, debido a que las altas temperaturas ambientales traen problemas en su manipulación y almacenamiento en lugares sin control de clima.

En Cuba se desarrolló un sellante dental, CUBRIDEM, el cual cumple con las normativas internacionales y mantiene su estabilidad como mínimo por un año, entre 2 y $8^{\circ} \mathrm{C}$. El objetivo de este trabajo ha sido obtener una variante de CUBRIDEM más estable a mayores temperaturas y con tiempos de trabajo (TT) y de fraguado (TF) adecuados, cuando el producto se manipula en ambientes no climatizados.

Se realizaron diseños experimentales factoriales $2^{3}$ donde se estudió la influencia de la concentración de los componentes minoritarios: peróxido de benzoílo (PBO), N,N-dimetil p-toluidina (DMPT) y éter monometílico de la hidroquinona (MHQ), manteniendo invariable la composición de la mezcla monomérica Bis-GMA/Dimetacrilato de tetraetilenglicol: 1/1.

El sellante, compuesto por un Diluyente que contiene $0.8 \%$ de PBO y $0.05 \%$ de MHQ y una Base con $0.8 \%$ DMPT, manipulado a $30 \pm 2{ }^{\circ} \mathrm{C}$ después de un almacenamiento por dos meses a la misma temperatura, permite obtener valores de TT y TF cercanos a los deseados. Por otra parte, el almacenamiento a $16 \pm 2{ }^{\circ} \mathrm{C}$ y un contenido de 0,6 \% de DMPT en la Base permiten lograr tiempos similares para almacenamientos más prolongados. Los resultados de las regresiones lineales sugieren que, para un almacenamiento a $16 \pm 2{ }^{\circ} \mathrm{C}$, contenidos de $0.7 \%$, tanto de PBO en Diluyente como de DMPT en Base, posibilitan alcanzar tiempos satisfactorios: $T T=44$ s y $T F=113 \mathrm{~s}$.
\end{abstract}

\section{Summary}

Dental sealant are used in dentistry to sealed the natural fissure of teeth and avoid the deposition of the streptococcus mutants which produce dental caries. Those materials are supplied in two portions, which polymerize when are mixed. Nevertheless, the use of these products in tropical countries could be difficult because high temperatures, during manipulation and storage, produce problems.

In Cuba a dental sealant, CUBRIDEM, was developed. The properties of this sealant agree with international standards and it remains stable by one year minimum, when stored at $2-8^{\circ} \mathrm{C}$. This work deals with the obtainment of a more stable CUBRIDEM, even transported and stored at not-refrigerated conditions, characterized by suitable periods of time for handling and setting.

Experimental work was carried out according to $2^{3}$ factorial designs, where the influence of minor components: benzoyl peroxide (PBO), N,N dimhetyl p-toluidine (DMPT) and hydroquinone monomethyl ether (MHQ) was studied while keeping monomeric composition unchanged (Bis-GMA/DMTEG: 1/1).

The formulation, which Diluent composition comprises $0.8 \% \mathrm{PBO}$ and $0.05 \% \mathrm{MHQ}$ whereas $D M P T$ content in Base is $0.8 \%$, handled at $(30 \pm 2)^{\circ} \mathrm{C}$ after two months of storage at the same temperature, allows the obtainment of handling and setting times close to the desired ones. By other side, storage at $(16 \pm 2)^{\circ} \mathrm{C}$ and a DMPT content of $0.6 \%$ in Base produces similar results but for longer time of storage. Results of linear regression suggest that control of PBO and DMPT contents, both at $0.7 \%$, leads to satisfactory handling $\quad(T T=44 \mathrm{~s})$ and setting $(T F=113 \mathrm{~s})$ times for formulations stored at $(16 \pm 2)^{\circ} \mathrm{C}$.

Keywords: Pit and fissure sealant, selfcured sealant, radicalic polymerization, storage, stability 


\section{Introducción}

El empleo de materiales poliméricos como sellantes de fosas y fisuras permite la prevención de la caries dental ${ }^{1}$. Estos productos están compuestos principalmente por Bis-GMA o monómero de Bowen, el cual es un compuesto de alto peso molecular y alta viscosidad. Se adicionan a los sellantes los llamados diluyentes, tales como el dimetacrilato tetraetilenglicol (DMATEG), los cuales permiten reducir la viscosidad de la mezcla ya que el producto debe ser lo suficientemente líquido para poder fluir al interior de las fisuras y mojar el diente, garantizándose una buena adaptación a su superficie ${ }^{2}$.

De acuerdo al mecanismo de polimerización, los sellantes pueden ser de dos tipos: autocurados y fotocurados ${ }^{3}$. Los autocurados se presentan en dos partes separadas. En una de ellas se encuentra el iniciador y en la segunda el acelerador de la polimerización radicálica. Al mezclar estas dos partes en el momento de la aplicación, comienza la polimerización. Los sellantes fotocurados son aquellos que polimerizan mediante la acción de la luz visible aplicada con una lámpara especial.

Los sellantes dentales autocurados polimerizan rápidamente por vía radicálica con la participación de iniciadores, como el peróxido de benzoílo (PBO). Por otra parte, cuando se requiere una polimerización rápida y a bajas temperaturas, se utilizan aceleradores de la polimerización que forman un par redox iniciador-acelerador. Una de las sustancias mas utilizadas en los materiales dentales, formando el par redox con el peróxido de benzoílo es la amina terciaria N,N-dimetil-ptoluidina (DMPT). También se conoce que, los dimetacrilatos polimerizan espontáneamente por vía radicálica, en las condiciones comunes de almacenamiento, a menos que estén presentes sustancias inhibidoras. Actualmente uno de los inhibidores más utilizados es el éter monometílico de la hidroquinona (MHQ) ${ }^{2}$.

Teniendo en cuenta la importancia que tiene garantizar la salud bucal y disminuir el nivel de caries en la población infantil, los sellantes denta-

\footnotetext{
Correspondencia:

Anastasia Mishina

Aseguramiento de la Calidad, Centro de

Biomateriales, Universidad de la Habana, Cuba

Fax: 335863

e-mail: nastia@biomat.uh.cu
}

les poseen una alta demanda en estomatología. Sin embargo, los sellantes autocurados y otros materiales monoméricos que polimerizan por vía radicálica, como son los cementos óseos acrílicos, presentan problemas durante su manipulación y almacenamiento en climas tropicales. Es por ello que el objetivo de este trabajo ha sido obtener una variante de CUBRIDEM, sellante dental cubano, más estable a mayores temperaturas y con tiempos de trabajo (TT) y de fraguado (TF) adecuados, cuando el producto se manipula en ambientes tropicales no climatizados.

\section{Materiales y métodos}

Para la preparación de los sellantes se utilizaron los reactivos siguientes:

- 2,2-bis-[p-(2-hidroxi-3-metacriloxipropoxi) fenil]propano (Bis-GMA) Nupol 046-4005 (FREEMAN),

- Dimetacrilato de tetraetilenglicol (FLUKA),

- Peróxido de benzoílo (ALDRICH, recristalizado en metanol),

- N,N dimetil p-toluidina (ALDRICH),

- Eter monometílico de la hidroquinona (FLUKA).

Se prepararon diferentes formulaciones del sellante, variando las concentraciones de los componentes minoritarios (PBO, DMPT y MHQ), manteniendo constante la composición monomérica Bis-GMA/DMATEG: 1/1 (p/p).

Se diseñó un primer bloque experimental $2^{3}$ en el cual se mantuvo fija la composición de la Base, donde a la mezcla monomérica se le adicionó 0.8 $\%$ de DMPT, y se realizaron variaciones a la formulación del Diluyente modificando las concentraciones de PBO y MHQ. Se estudió el efecto de las variables independientes sobre las respuestas después de un almacenamiento a temperaturas entre 14 y $18^{\circ} \mathrm{C}$ y entre 28 y $32^{\circ} \mathrm{C}$.

Las variables independientes estudiadas fueron las siguientes:

- $\mathrm{X}_{1}$ : Concentración de PB en el Diluyente $(0.6 \%$ y $0.8 \%)$,

- $\mathrm{X}_{2}$ : Concentración de MHQ en el Diluyente $(0.03 \%$ y $0.05 \%)$

- $\mathrm{X}_{3}$ : Temperatura de almacenamiento $\left(16 \pm 2^{\circ} \mathrm{C}\right.$ y $\left.30 \pm 2^{\circ} \mathrm{C}\right)$.

Mientras que las respuestas a analizar fueron:

- $\mathrm{Y}_{1}$ : tiempo de trabajo (TT),

- $\mathrm{Y}_{2}$ : tiempo de fraguado (TF). 
Se realizaron también experimentos adicionales en este bloque con las siguientes condiciones: $0.7 \%$ de PBO, $0.04 \%$ de MHQ y almacenamiento a las dos temperaturas del diseño. La estimación del error puro se realizó utilizando los resultados de tres réplicas de las muestras en cada punto experimental.

Se realizó un segundo bloque experimental $2^{3}$ donde se sustituyó la variable temperatura de almacenamiento por la dosis de DMPT en la Base, la cual se varió en dos niveles: $0.6 \%$ y $0.8 \%$.

Para la aplicación clínica del material, es de gran importancia que exista un tiempo suficiente entre el momento en que se comienzan a mezclar las partes y la aplicación del producto sobre la superficie del diente, durante el cual no se incremente excesivamente la viscosidad, ya que este incremento ocasiona que disminuya la penetración del material en las fosas y fisuras. Este período se conoce como tiempo de trabajo. Por otra parte, una vez colocado el material, este debe fraguar lo más rápido posible, conociéndose como tiempo de fraguado aquel desde el momento en que se comienzan a mezclar las partes hasta que el producto endurece.

Los tiempos de trabajo y fraguado fueron determinados a la temperatura ambiente $\left(30 \pm 2^{\circ} \mathrm{C}\right)$ por un método de observación visual que simula la manipulación que realiza el estomatólogo en el momento de aplicar el sellante. Se añade una gota de cada una de las partes en un pozuelo de aplicación. Se pone en marcha el cronómetro cuando se añade la segunda gota. Se homogeneiza la mezcla con un explorador dental de cucharilla durante 10 segundos. Posteriormente se introduce la punta del explorador en la mezcla a pequeños intervalos hasta que al retirarlo aparece un hilo fino de resina entre éste y la mezcla. Este momento se toma como TT. Para la determinación del TF se utiliza un explorador de punta fina, que se introduce suavemente en la mezcla a cortos intervalos. Se toma como TF aquel cuando la dureza del material impide la penetración del explorador.

\section{Resultados y discusión}

La norma ISO 6874:1988 establece los tiempos de trabajo y fraguado óptimos, para este tipo de producto, determinados a $23^{\circ} \mathrm{C}$ y $37^{\circ} \mathrm{C}$ respectivamente. El tiempo de trabajo se establece como 45 $\mathrm{s}$, mientras que el tiempo de fraguado no debe exceder de $5 \mathrm{~min}^{4}$. Sin embargo, a la temperatura ambiente de Cuba $\left(28-32^{\circ} \mathrm{C}\right)$ la velocidad de polimerización es mayor que a $23^{\circ} \mathrm{C}$, lo que acorta el tiempo real de trabajo. Por esta razón, el objetivo de esta investigación ha sido lograr tiempos de trabajo cercanos a los 45 segundos pero a la temperatura ambiente de Cuba, por lo que en todos los experimentos el mezclado de Base con el Diluyente se realizó a esta temperatura $\left(28-32^{\circ} \mathrm{C}\right)$. Los resultados del primer diseño experimental se muestran en la Tabla 1.

Se observa que todas las formulaciones almacenadas por tres meses a temperatura ambiente $\left(30 \pm 2^{\circ} \mathrm{C}\right)$ presentan tiempos de trabajo y fraguado excesivamente largos. Este comportamiento se puede apreciar también en la figura 1. En la misma se muestran los resultados obtenidos para una de las formulaciones a las dos temperaturas de almacenamiento (Exp. 4 y 8). Los tiempos de trabajo y fraguado después de almacenamiento a temperaturas entre 28 y $32^{\circ} \mathrm{C}$ (TTC y TFC) presentaron aumentos significativos después de dos meses, lo que limita el tiempo máximo de almacenamiento a temperatura ambiente para estas formulaciones.

Sin embargo, para las muestras almacenadas a temperaturas entre 14 y $18^{\circ} \mathrm{C}$ los tiempos (TTF y TFF) se han mantenido estables durante los tres meses. Con la excepción del Exp. 3, cuyos tiempos

Tabla 1. Resultados del diseño experimental realizado con un contenido de DMPT de $0.8 \%$.

\begin{tabular}{|c|c|c|c|c|c|c|c|c|c|}
\hline Exp. & \multicolumn{3}{|c|}{ Variables independientes } & \multicolumn{5}{c|}{ Resultados } \\
\hline & PBO & MHQ & Temp. alm. & \multicolumn{3}{c|}{ TT } & \multicolumn{3}{c|}{ TF } \\
\hline & & & & 7 días & 2 meses & 3 meses & 7 días & 2 meses & 3 meses \\
\hline 1 & $0.6 \%$ & $0.03 \%$ & $16 \pm 2{ }^{\circ} \mathrm{C}$ & $28 \pm 2$ & $28 \pm 2$ & $38 \pm 4$ & $89 \pm 4$ & $85 \pm 4$ & $91 \pm 5$ \\
\hline 2 & $0.8 \%$ & $0.03 \%$ & $16 \pm 2^{\circ} \mathrm{C}$ & $19 \pm 2$ & $21 \pm 2$ & $25 \pm 3$ & $57 \pm 4$ & $58 \pm 5$ & $55 \pm 5$ \\
\hline 3 & $0.6 \%$ & $0.05 \%$ & $16 \pm 2^{\circ} \mathrm{C}$ & $37 \pm 4$ & $40 \pm 2$ & $52 \pm 3$ & $138 \pm 10$ & $145 \pm 10$ & $150 \pm 10$ \\
\hline 4 & $0.8 \%$ & $0.05 \%$ & $16 \pm 2^{\circ} \mathrm{C}$ & $26 \pm 1$ & $26 \pm 3$ & $28 \pm 3$ & $85 \pm 4$ & $70 \pm 5$ & $75 \pm 5$ \\
\hline 5 & $0.6 \%$ & $0.03 \%$ & $30 \pm 2^{\circ} \mathrm{C}$ & $28 \pm 4$ & $60 \pm 3$ & $>180$ & $91 \pm 9$ & $120 \pm 9$ & N/M \\
\hline 6 & $0.8 \%$ & $0.03 \%$ & $30 \pm 2^{\circ} \mathrm{C}$ & $20 \pm 2$ & $28 \pm 2$ & $80 \pm 5$ & $60 \pm 5$ & $65 \pm 6$ & $165 \pm 11$ \\
\hline 7 & $0.6 \%$ & $0.05 \%$ & $30 \pm 2^{\circ} \mathrm{C}$ & $37 \pm 5$ & $>180$ & N/M & $143 \pm 13$ & $>300$ & N/M \\
\hline 8 & $0.8 \%$ & $0.05 \%$ & $30 \pm 2^{\circ} \mathrm{C}$ & $26 \pm 1$ & $35 \pm 3$ & $>120$ & $85 \pm 4$ & $90 \pm 5$ & $>180$ \\
\hline 9 & $0.7 \%$ & $0.04 \%$ & $16 \pm 2^{\circ} \mathrm{C}$ & $26 \pm 1$ & $27 \pm 3$ & $33 \pm 3$ & $86 \pm 4$ & $80 \pm 5$ & $85 \pm 5$ \\
\hline 10 & $0.7 \%$ & $0.04 \%$ & $30 \pm 2^{\circ} \mathrm{C}$ & $26 \pm 3$ & 50 & $>120$ & $90 \pm 10$ & $113 \pm 8$ & $>180$ \\
\hline
\end{tabular}




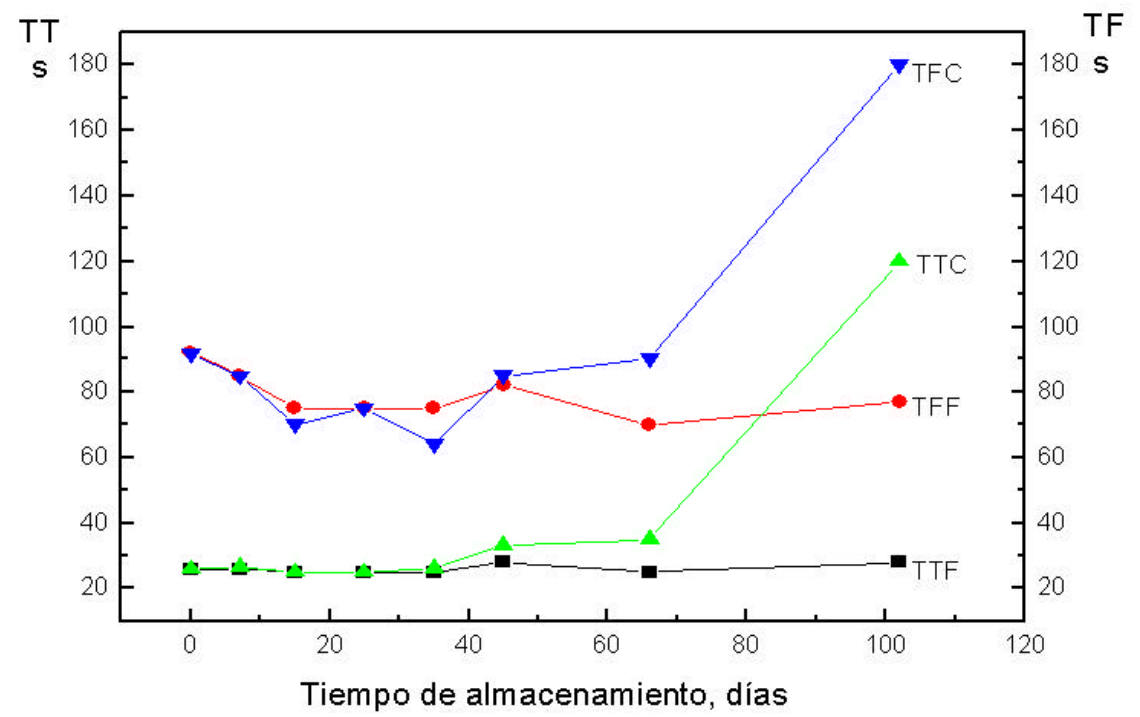

Figura 1. Variación de TT y TF durante el almacenamiento a diferentes temperaturas TTC y TFC-tiempos de trabajo y fraguado para muestras almacenadas a $28-32{ }^{\circ} \mathrm{C}$ TTF y TFF-tiempos de trabajo y fraguado para muestras almacenadas a $14-18{ }^{\circ} \mathrm{C}(0.8 \%$ de PBO, $0.05 \%$ de MHQ y $0.8 \%$ de DMPT $)$.

de fraguado son excesivos prácticamente desde el inicio, el resto de las formulaciones a $16 \pm 2^{\circ} \mathrm{C}$ presenta TT algo más cortos que los requeridos para la manipulación del producto por el estomatólogo, por lo que se probó una nueva formulación donde la Base se preparó con $0.6 \%$ de DMPT, manteniendo la misma composición del Diluyente. Esto originó el segundo bloque $2^{3}$, según se muestra en la tabla 2 .

Estos resultados fueron procesados por regresión lineal, lo que permitió obtener modelos cuyos parámetros de ajuste se muestran en la tabla 3. Estos modelos mostraron un excelente ajuste, evidenciado por los coeficientes de correlación superiores a los valores críticos, aún para niveles de significación $(\alpha)$ de 0.98 .

Del análisis de estas ecuaciones se observa que la concentración de DMPT $\left(\mathrm{X}_{3}\right)$ ejerce la mayor influencia sobre TTF, así como que se obtienen mayores valores de esta respuesta para el nivel máximo de MHQ $\left(\mathrm{X}_{2}\right)$. En la figura 2 se ilustra el comportamiento de TTF versus DMPT a diferentes ni-

Tabla 2. Resultados obtenidos para dos meses de almacenamiento entre 14 y $18^{\circ} \mathrm{C}$.

\begin{tabular}{|c|c|c|c|c|}
\hline PBO $\left(\mathbf{X}_{\mathbf{1}}\right)$ & MHQ $\left(\mathbf{X}_{\mathbf{2}}\right)$ & DMPT $\left(\mathbf{X}_{\mathbf{3}}\right)$ & TTF & TFF \\
\hline $0.6 \%$ & $0.03 \%$ & $0.6 \%$ & 53 & 135 \\
\hline $0.8 \%$ & $0.03 \%$ & $0.6 \%$ & 32 & 140 \\
\hline $0.6 \%$ & $0.05 \%$ & $0.6 \%$ & 75 & 190 \\
\hline $0.8 \%$ & $0.05 \%$ & $0.6 \%$ & 38 & 105 \\
\hline $0.6 \%$ & $0.03 \%$ & $0.8 \%$ & 28 & 85 \\
\hline $0.8 \%$ & $0.03 \%$ & $0.8 \%$ & 21 & 58 \\
\hline $0.6 \%$ & $0.05 \%$ & $0.8 \%$ & 38 & 120 \\
\hline $0.8 \%$ & $0.05 \%$ & $0.8 \%$ & 25 & 70 \\
\hline
\end{tabular}

veles del PBO.

En la figura 2 se evidencia que a medida que aumenta el contenido de PBO disminuye el tiempo de trabajo después del almacenamiento a $14-18^{\circ} \mathrm{C}$. Por lo tanto, niveles máximos de $\mathrm{PBO}$ producen valores de TTF por debajo de lo deseado. Para el nivel medio de PBO y DMPT (ambos $0.7 \%$ ) y máximo de MHQ (0.05\%), se logra un valor de TTF que satisface las expectativas planteadas (44 s). Para estas condiciones se pronostica un TFF de 113 segundos. La comprobación de estos resultados será objeto de trabajos posteriores.

\section{Conclusiones}

El tiempo máximo de almacenamiento de las formulaciones estudiadas a $30 \pm 2{ }^{\circ} \mathrm{C}$ es de dos meses, el cual puede extenderse a periodos más prolongados a $16 \pm 2{ }^{\circ} \mathrm{C}$. La concentración de N,N dimetil p-toluidina ejerce la mayor influencia sobre los tiempos de trabajo y de fraguado para el almacenamiento a $16 \pm 2{ }^{\circ} \mathrm{C}$. Para el nivel medio $(0.7$ $\%)$ de peróxido de benzoílo y máximo $(0.05 \%)$ de éter monometílico de la hidroquinona, el tiempo de trabajo puede alcanzar un valor de $44 \mathrm{~s}$ cuando

Tabla 3. Ajuste estadístico de modelos.

\begin{tabular}{|l|c|c|}
\hline \multirow{2}{*}{ Parámetro } & M O D E L O & A J U S T A D O \\
\cline { 2 - 3 } & TTF & TFF \\
\hline Coeficiente de correlación $(\mathrm{R})$ & 0.955 & 0.906 \\
\hline Grados de libertad $(\mathrm{f})$ & 3 & 4 \\
\hline Nivel de significación $(\alpha)$ & 0.98 & 0.98 \\
\hline R crítico $(\mathrm{f}, \alpha) \quad 0.934$ & 0.882 \\
\hline \multicolumn{2}{|c|}{ Modelos TTF $=38.75-9.75 \mathrm{X}_{1}+5.25 \mathrm{X}_{2}-10.75 \mathrm{X}_{3}+4.75 \mathrm{X}_{1} \mathrm{X}_{3}$} \\
$\mathrm{TFF}=112.875-19.625 \mathrm{X}_{1}-29.625 \mathrm{X}_{3}-14.125 \mathrm{X}_{1} \mathrm{X}_{2}$
\end{tabular}




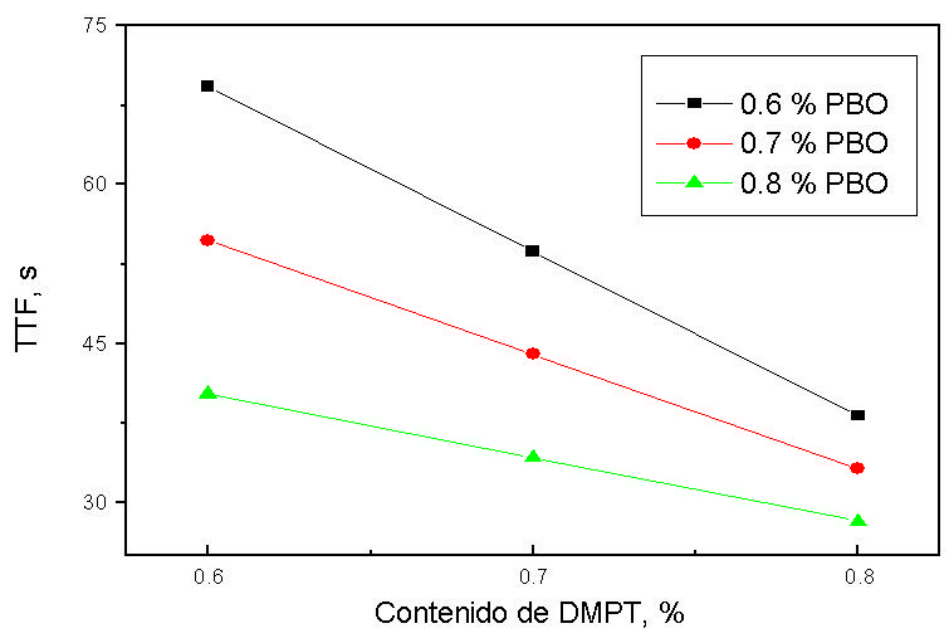

Figura 2. Influencia de la concentración del DMPT sobre el TTF a diferentes niveles de PBO para $0.05 \%$ de MHQ.

el contenido de N,N-dimetil p-toluidina sea $0.7 \%$. Para estas condiciones el tiempo de fraguado será de 113 segundos.

\section{Referencias}

1. Rodríguez Miró, M.J. Etiopatogenia de la Caries Dental. Actualidad en Estomatología. 1979; 1: 3.

2. Bowen, R.L Compatibility of various materials with oral tissues. I. The components in composite restaurations. Journal Dental Research. 1979:58 (5):1493-1503.

3. Phillips, R.W., Science of Dental Materials, Ed. 8, W.B. Saunders Company, (1982).

4. ISO 6874:1988 Dental resin-based pit and fissure sealants. 